\title{
Philosophiques
}

\section{Leibniz sur l'espace, le continu et la substance : mathématique, physique et métaphysique \\ François Duchesneau, La dynamique de Leibniz, Paris, Vrin, coll. Mathesis, 1994}

\section{Luciano Boi}

Volume 22, numéro 2, automne 1995

URI : https://id.erudit.org/iderudit/027344ar

DOI : https://doi.org/10.7202/027344ar

Aller au sommaire du numéro

Éditeur(s)

Société de philosophie du Québec

ISSN

0316-2923 (imprimé)

1492-1391 (numérique)

Découvrir la revue

Citer cet article

Boi, L. (1995). Leibniz sur l'espace, le continu et la substance : mathématique, physique et métaphysique / François Duchesneau, La dynamique de Leibniz, Paris, Vrin, coll. Mathesis, 1994. Philosophiques, 22(2), 407-436.

https://doi.org/10.7202/027344ar d'utilisation que vous pouvez consulter en ligne. 


\title{
LEBBNIZ SUR L'ESPACE, LE CONIINU EI LA SUBSTANCE : MATHÉMATIQUE, PHYSIOUE ET MÉTAPHYSIQUE*
}

\author{
par Luciano Boi
}

\section{L'intérêt et la signification de l'euvre de Leibniz}

Dès le début, la philosophie et la science de Leibniz ont inspiré le travail des philosophes, des mathématiciens et des physiciens. Tout comme Aristote, Descartes, Newton, Kant, Husserl ou Einstein, Leibniz a su soulever des questions tout à fait incontournables pour tous ceux qui ont cherché à mieux comprendre les rapports intimes qui lient la pensée mathématique aux théories physiques, d'une part, et ces mêmes théories aux idées philosophiques et principes métaphysique, d'autre part. L'intérêt porté à l'ceuvre de Leibniz se poursuit encore aujourd'hui : la preuve en est l'ouvrage récent que François Duchesneau consacre à l'cuvre majeure du philosophe, la Dynamique . En plus de procéder à une reconstruction historique de ce corpus de textes et d'essayer de donner une nouvelle interprétation de leur signification et de leur portée épistémologiques, l'auteur nous livre une analyse des plus complètes des faits majeurs qui ont conduit Leibniz à l'élaboration d'une nouvelle science physique. Les résultats d'une telle analyse sont d'autant plus remarquables que François Duchesneau contribue à mettre en évidence les nouveaux points de vue méthodologiques et épistémologiques qui se dégagent de l'ceuvre de Leibniz, et dont la prise en considération est essentielle, selon l'auteur, pour une compréhension véritable de son travail proprement scientifique.

Certes, un commentateur pressé, et en plus se réclamant d'une vision foncièrement positiviste de la science, pourrait affirmer qu'après tout, ce qui est resté des idées de Leibniz sur la physique est bien peu de chose comparé à l'édifice de la mécanique galiléenne-newtonienne. Et même en ce qui concerne la dynamique, ce serait la mécanique analytique telle qu'elle a été développée par Lagrange et puis par Hamilton que nous appliquerions de nos

* Nous tenons à remercier ici F. Duchesneau et R. Nadeau d'avoir bien voulu relire la version définitive de ce texte.

1. F. Duchesneau, La dynamique de Leibniz, Paris, Vrin, 1994. Cf. également son autre ouvrage portant sur Leibniz, Leibniz et la méthode de la science, Paris, P.U.F., 1993. 
jours, plutôt que les théories plus ou moins scientifiques de Leibniz. Or personne n'aurait de mal à voir le caractère par trop partial et injuste d'un tel jugement. Il est vrai que la mécanique de Newton donnant les lois mathématiques du mouvement des corps solides dans un système de référence spatiotemporel euclidien-galiléen a été corroborée par l'expérience, et que sa loi de gravitation terrestre et céleste, fondée sur la notion d' " action à distance ", est en général confirmée expérimentalement. Rappelons ici, en particulier, que l'équation fondamentale donnée par Newton dans ses Principia mathematica ${ }^{2}$, exprimant la force comme le produit de la masse d'un point matériel et de son accélération : $F=m \gamma$, où la vitesse est par définition le vecteur $\gamma=\frac{d r}{d t}-r$ désignant le point géométrique-matériel pris dans le système inertiel de coordonnées ou espace affine quadridimensionnel $\boldsymbol{R}^{4}$ (i.e. «Univers de Galilée ») - garde toujours sa validité, et que, de ce fait, la loi de la gravitation de Newton a été incorporée comme un cas limite dans les théories relativistes qui lui ont succédé. On ne saurait pourtant méconnaître les limites intrinsèques des conceptions de Newton, car c'est précisément à partir de la critique de ces deux paradigmes de la science newtonienne que Maxwell a pu concevoir une théorie dynamique des forces physiques ou du chanp électromagnétique, et qu'Einstein a élaboré les théories de la relativité restreinte et générale. Ces deux réalisations majeures de la physique, ont non seulement permis un dépassement des théories de Newton auxquelles nous avons fait allusion, mais encore ont-elles remis définitivement en question le point de vue épistémologique qui leur avait servi de fondement, et notamment l'idée de l'espace et du temps absolus.

Rappelons succinctement en quoi cette transformation conceptuelle a consisté. La science galiléenne-newtonienne était essentiellement fondée sur la considération d'un univers purement mécanico-cinématique formé de points matériels dont les mouvements effectifs et virtuels, de même que les lois auxquelles ils obéissaient, étaient censés être parfaitement décrits au moyen d'une représentation géométrique et par des systèmes d'équations et de substitutions analytiques invariantes. Ces points étaient évidemment considérés comme étant dépourvus de forces et de toute autre propriété physique de nature dynamique. Maxwell remplace cet univers abstrait et physiquement latent par le concept de champ, qui seul permet d'expliquer certaines propriétés physiques affectant les corps (et précisément les forces électriques et magnétiques qui, chez le physicien écossais, se trouvent pour la première fois ramenées au même principe) au voisinage d'un point donné ou d'une certaine région de l'espace. Le concept de champ est également lié à l'idée qu'il doit exister une substance impondérable qu'est l'éther permettant la propagation des effets

2. I. Newton, Philosophia naturalis principia mathematica (1687)/Mathematical Principles of Natural Philosophy, trad. A. Motte, éd. révisée par F. Cajori, Berkeley, University of Califomia Press, 1962. 
physiques réciproques d'un corps à l'autre dans l'espace vide. On voit donc que, malgré la reconnaissance d'une interaction effective entre les corps physiques et la région d'espace avoisinante, le champ n'est pas encore conçu comme un concept proprement physique, mais plutôt comme un agent des corps affectés de certains états électriques et magnétiques, sans pour autant qu'il soit lui-même pourvu de propriétés dynamiques. Cela n'empêche pas que Maxwell ait donné les equations du mouvement des corps sujets à des forces électriques et magnétiques dans le vide.

Pour notre analyse ultérieure de la pensée de Leibniz à partir de l'ouvrage de Duchesneau, il importe de souligner encore les points suivants :

i) la théorie électromagnétique de Maxwell est fondée sur la notion d' «action de contact », c'est-à-dire sur un principe de continuité physicogéométrique ; autrement dit, elle fait intervenir l'idée que la structure géométrique locale de l'espace et les propriétés physiques des corps doivent en quelque sorte coexister ;

ii) cette même théorie émancipe la notion de force de toute conception mécaniste en écartant définitivement l'idée de solidité et d'impénétrabilité des corps ainsi que les autres notions qui leur sont reliées. Dans ce contexte, l'introduction de la notion d'éther (une substance fluide et subtile imprégnant l'espace tout entier) a joué un rôle très important, car elle a permis d'expliquer le phénomène des actions physiques réciproques entre les corps ;

iii) selon la théorie en question, un champ, bien qu'il soit dépourvu de toute masse pondérable, est rempli de matière éthérale, et c'est ce qui assure, pour ainsi dire, une coexistence des forces physiques avec ces parties de l'espace où elles sont produites. On peut dire ainsi que la théorie électromagnétique de Maxwell est la première théorie dynamique des forces physiques fondamentales. C'est Maxwell lui-même qui souligne, avec une clarté remarquable, ce qu'on doit entendre par là :

[...] la théorie que je propose peut être appelée une théoric du champ électromagnétique, parce qu'elle a à voir avec l'espace ì proximité des corps électriques et magnétiques, et une théorie dynamiqué, parce qu'elle admet que dans cet espace il y a de la matière en mouvement, grâce à laquelle les phénomènes électromagnétiques observés sont produits. Le champ électromagnétique est cette partie de l'espace qui contient et entoure les corps dans leur état électrique ou magnétique. Il peut être rempli de toutes sortes de matière; nous pouvons essayer aussi de le vider de toute matière pondérahle, comme dans le cas des tuhes de Geissler et autres soi-disant vides. Il reste toujours cependant assez de matière pour recevoir et transmettre les ondulations de la lumière et de la chaleur, et c'est parce que la transmission de ces radiations n'est pas altérée de manière sensible lorsque des corps transparents de densité mesurable se substituent au soi-disint vide, que nous sommes obligés d'admettre que les ondulations sont celles d'une substance de la nature de l'éther, et non pas de la matière pondérable, dont la présence modifie tout simplement d'une façon ou d'une uutre le mouvement de l'éther. Nous avons 
donc tout lieu de croire, d'après des phénomènes de la lumière et de la chaleur, qu'il existe un milieu ayant la nature de l'éther, remplissant l'espace et pénétrant les corps, capable d'être mis en mouvement et de transmettre ce mouvement d'un lieu à un autre, ainsi que de le communiquer à la matière pondérable en vue de la réchauffer et de l'affecter de différentes façons ${ }^{3}$.

Grâce à la théorie de la relativité générale, Einstein réussit à compléter de façon essentielle et décisive la conception du physicien-théoricien écossais. Il montre que les forces d'inertie et gravitationnelle, au lieu d'être de nature différente, sont en fait la même force qui ne se manifeste différemment que du point de vue phénoménologique ; par conséquent, elles peuvent être expliquées selon les mêmes principes théoriques ou mathématiques. Il propose ainsi l'unification de ces deux forces. Mais ce qui est plus fondamental encore dans la conception d'Einstein, comme Hermann Weyl a tenu à le souligner ${ }^{4}$, est que le champ métrique tire sa propre existence d'une propriété profonde de l'univers, à savoir de sa structure géométrique infinitésimale. Alors que dans la physique classique, le champ métrique est une forme géométrique en soi appartenant à l'univers et indépendante de la matière qui la remplit et des forces qui l'animent, pour Einstein, le champ (et son état) a une réalité physique

3. Cf. J. C. Maxwell, «A Dynamical Theory of the Electromagnetic Field » (1864), in The Scientific Papers of Iames Clerk Maxwell (1 ${ }^{\text {re }}$ éd., 1890), Dover, New York, 1965 , p. 526-528.

4. Cf. H. Weyl, « Feld und Materie », Annalen der Physik, 65 (1921), 541-563. Voir également l'ouvrage classique de l'auteur, Space-Time-Matter ( $1^{\text {re }}$ éd. en all., 1918), Dover, New York, 1922, en part. le chap. IV, \& 29 : « Relativité du mouvement, champ métrique et gravitation $"$. Ici, l'auteur rappelle d'abord que, dans la théorie de la relativité restreinte d'Einstein, les mêmes phénomènes physiques demeurent valables dans n'importe quel système de référence arbitrairement donné, mais cela vaut uniquement d'un point de vue cinématique. Il n'en est pas de même lorsqu' on considère les effets dynamiques des phénomènes physiques. Il se pose alors la question, par exemple, de savoir « où se trouve l'origine de la force centrifuge et des autres forces d'inertie? "Dans l'espace absolu, répondait Newton. La réponse que donne la relativité restreinte n'est pas au fond différente : ces phénomènes sont dus à la structure métrique de l'univers considérée comme une propriété géométrique formelle. « Mais ce qui engendre la force, écrit Weyl, doit aussi possécler une réalité. Or, la seule réalité que nous puissions admettre pour cela, c'est la structure métrique ; elle devrait être elle-même susceptible de variations et pouvoir subir les effets de la matière. [...] L'existence d'une géométrie indépendante de la physique est définitivement compromise... [...] En vérité, les mesures spatiales reposent sur un phénomène physique : l'action des rayons lumineux et des règles de mesure sur l'ensemble de l'univers matériel. [...] La manière d'agir des rayons lumineux ou les effets sur les règles de mesure sont déterminés, par leurs propriétés intrinsèques bien entendu, mais, en outre, par le champ métrique (ou, mathématiquement parlant, par le tenseur $g_{i k}$ ) précisément comme la manière d'agir d'une charge électrique est déterminée par le champ électrique et par les propriétés intrinsèques de cette charge. Mais, de même que le champ électrique dépend des charges, nous devons admettre que le champ métrique dépend des réalités matérielles qui remplissent l'univers. " 
qui se trouve en échange réciproque avec la matière. Dans sa théorie, le champ métrique embrasse le phénomène de la gravitation. Par exemple, le mouvement des planètes suit une trajectoire qui leur est prescrite en quelque sorte par le champ métrique : à l'encontre de ce que pensait Newton, il ne peut donc y avoir de «force de gravitation » nécessaire et absolue (agissant à distance) qui dévierait d'un tel mouvement. La séparation du champ métrique en deux composantes, l'inertie et la gravitation, apparaît ainsi arbitraire et ne peut pas avoir de signification objective.

Bref, le caractère révolutionnaire et la signification épistémologique profonde de la relativité générale résident dans le fait d'avoir conçu le champ métrique (auquel la gravitation se trouve finalement ramenée) dans un rapport causal avec la matière et ses forces. En des termes plus généraux, Einstein a montré qu'on ne peut pas séparer la structure géométrique de l'univers de la matière et donc des phénomènes physiques qui se déroulent en lui : les effets gravitationnels dans l'univers sont à attribuer aux modifications de la géométrie de l'espace-temps. À la base de la relativité générale se trouve ainsi le nouveau concept d' "espace-temps ", conçu désormais comme une variété pseudo-riemannienne affine à 4 dimensions dans laquelle chaque entité géométrique déterminée (comme, par exemple, un " point ", une " géodésique ", une « connexion », etc.) exprime un cerlain événement physique (un rayon de lumière, par exemple). À cela il faut ajouter le rôle considérable joué par la conception de l'éther comme un état de l'espace physique lui-même, doué de propriétés dynamiques mais dépourvu de toute signification mécanique et cinématique.

Or, il n'y a pas de doute que Leibniz ait été l'un des précurseurs de cette transformation radicale des conceptions de la matière et de l'espace dans la physique, bien qu'il n'en ait pas été directement à l'origine. Sans vouloir parler ici d'anticipation, ce qui pourrait paraître plutôt arbitraire, il y a lieu de penser que, par l'édification de sa Dynamique, Leibniz a contribué à ce que les conceptions de Maxwell et d'Einstein mentionnées plus haut voient le jour et se développent. En effet, on trouve dans plusieurs passages décisifs de la Dynamique ainsi que dans d'autres textes qui lui sont aussi bien antérieurs que postérieurs, non seulement des intuitions intéressantes, mais encore des idées essentielles dont on ne peut que souligner les points communs avec celles qui serviront, un siècle plus tard, de fondement aux théories de l'électromagnétisme et de la relativité générale. Concentrons-nous sur une des conceptions de Leibniz qui, en plus d'apparaître fondamentale pour comprendre l'cuvre du philosophe en général, sert à appuyer la thèse que nous venons tout juste de présenter. 


\section{La critique par Leibniz du programme mécaniste de Descartes et la constitution de la dynamique}

À l'encontre de Descartes, Leibniz ne considérait pas l'extension comme une propriété essentielle des corps. Il pensait que les propriétés géométriques d'étendue des corps (c'est-à-dire la longueur, la largeur et la profondeur) ne suffisent pas à en caractériser les propriétés physiques, d'où sa recherche d'autres principes qui pourraient être aptes à rendre compte des forces inhérentes aux phénomènes physiques qui se manifestent dans un "état actif », c'est-à-dire de «non repos». Une telle recherche, comme on le sait, aboutira dans la formulation complète du principe de conservation de la force vive (ou de l'action motrice), qui nous dit que les distances parcourues par un corps en mouvement et ayant acquis de la force vive, ne sont point proportionnelles aux vitesses, mais aux carrés des vitesses ; les forces vives ne sont donc pas comme les quantités de mouvement ou comme les produits des masses par les vitesses. Nous reviendrons tout à l'heure sur la signification et l'importance de ce théorème qui constitue le résultat fondamental de la Dynamique leibnizienne.

Or ces principes ne sauraient être de nature uniquement mathématique ou exprimables au moyen de simples concepts géométriques, car, pour pouvoir rendre compte des forces affectant les corps réels, il faut de plus qu'ils expriment un contenu physique véritable. Une telle conception, propre à Descartes, se heurterait à une contradiction d'ordre philosophique ainsi qu'à une disparité sur le plan propre même de l'explication physique. Par exemple, elle est incapable de donner une explication cohérente des mouvements de choc ou des mouvements contraints. Il s'agit ici évidemment de la grande confrontation opposant Leibniz à la conception mécanique de la nature et au système philosophique général de Descartes, et qui constitue un des épisodes-clés permettant de saisir les raisons profondes de l'établissement par Leibniz d'une théorie dynamique des phénomènes naturels et de comprendre le développement qui s'ensuit d'une nouvelle conception épistémologique de la physique.

Il est vrai par ailleurs que l'interprétation du programme cartésien est assez controversée, tout comme l'est celle de Leibniz. Certains interprètes ont voulu mettre l'accent sur le fait que Descartes aurait été le premier à concevoir un véritable programme de géométrisation de la physique ${ }^{6}$, ce qui paraîtrait quelque peu exagéré. D'autres savants ont insisté davantage sur sa vision mécaniste générale englobant à la fois les propriétés mécaniques et géométriques des corps, un point de vue qui pourrait sembler plutôt partiel. D'une manière générale, on s'accorde à reconnaître que Descartes a été d'abord et avant

5. Cf. R. Descartes, Euvres de Descartes, éd. par C. Adam et P. Tannery, nouv. présentation par B. Rochot et P. Costabel, Paris, Vrin, 1964-1974.

6. Cf. J. C. Graves, The Conceptual Foundations of Contemporary Relativity Theory, Cambridge, The MIT Press, 1971 ; voir p. 79-100. 
tout l'inventeur de la géométrie analytique et que par l'édification de cette nouvelle science des mathématiques il visait à exprimer toutes les notions et relations géométriques au moyen d'un langage algébrique le plus clair et simple possible, fondé sur des règles de transformation des équations linéaires jusqu'à un certain ordre et sur le système des coordonnées, ce qui historiquement représente un mérite mathématique indéniable. On ne peut pas dire cependant que Descartes ait prétendu que toutes les propriétés physiques des corps pouvaient être réduites à de simples notions et relations géométriques entre ces corps, et que cette réduction n'avait qu'un caractère symbolique et abstrait. Ses travaux sur des problemes scientifiques précis, comme ceux en optique, dioptrique, sur la lumière, etc., montrent qu'il concevait la géométrie comme étant également apte à foumir des modèles explicatifs des phénomènes physiques concernés. Il y a à ce propos, dans les travaux scientifiques de Descartes, une part d'intuition et d'analyse " qualitative » des phénomènes qui ne saurait être négligée. Vouloir essayer à tout prix de ramener son cuvre à la conception générale analytique et abstraite qu'il eut de la géométrie est une opération peu convaincante et douteuse'. L'idéal analytique et quantitatif appartient davantage à la science telle que développée par Newton qu'à la science pratiquée et défendue par Descartes ${ }^{8}$. On notera à ce propos que, selon Descartes, les concepts géométriques permettent d'établir une «physique $a$ priori » qui relie explicitement les prédictions expérimentales au rôle stratégique joué par certaines "grandeurs » mathématiques dans la constitution de l'espace physique. Il n'en demeure pas moins que, pour Descartes, toutes les propriétés physiques des corps sont de nature mécanique (c'est bien pour cela qu'il n'est question chez lui que de solidité et d'impénétrabilité), qui, en dernière analyse, peuvent être réduites à quelques propriétés géométriques. Une fois que l'étendue a été reconnue comme la propriété primordiale des corps physiques, c'est le mouvement qui doit exprimer la liaison entre ces

7. Cf. la postface de M. Tripp en annexe à l'édition en allemand de l'ouvrage de Descartes, Le Monde ou Traité de la Lumière/Die Welt oder Abhandlung über das Licht, tradl. par G. M. Tripp, Akademie Verlag, Weinheim, 1989, p. 143-166. Pour une étude approfondie des conceptions de Descartes à l'ceuvre dans sa métaphys ique et dans sa géométrie, of. L. Brunschvicg, Les étapes de la philosophie mathématique (1912), nouv. éd., Paris, A. Blanchard, 1981, en particulier les chapitres VII et VIII

8. Voir à ce propos la réflexion faite par R. Thom dans Stabilité structurelle et morphogenèse (Paris, InterÉditions, 1977, p. 5) : «Une autre cause de Ia défiance du physicien à l'égard du qualitatif est d'origine historique ; à la fin du XVIn ${ }^{\mathbf{e}}$ siècle, la controverse faisait rage entre tenants des physiques de Descartes et de Newton. Descartes, avec ses tourbillons, ses atomes crochus, etc. expliquait tout et ne calculait rien; Newton, avec la loi de gravitation en $1 / \mathrm{r}^{2}$, calculait tout et n'expliquait rien. L'histoire a donné raison à Newton et relégué les constructions cartésiennes au rang des imaginations gratuites et des souvenirs de musée. Certes, le point de vue newtonien se justifie pleinement du point de vue de l'efficacité, des possibilités de prédiction, donc d'action sur les phénomènes. " 
mêmes corps et les propriétés géométriques, et donc par une pure réduction cinématique.

Après avoir cherché à réformer le programme mécaniste de Wallis, Descartes, Mariotte et Huygens, Leibniz réalise la nécessité de constituer une nouvelle science physique, la Dynamique, pour expliquer un certain nombre de phénomènes qui lui paraissaient inintelligibles du point de vue de la mécanique cartésienne. Une telle ceuvre était accompagnée de la mise en place d'un programme méthodologique et épistémologique capable d'assurer les nouveaux fondements conceptuels de la science physique qu'il allait développer. Dans son ouvrage, La dynamique de Leibniz, non seulement François Duchesneau met-il très bien en évidence l'importance de ces points, mais encore montre-t-il qu'ils sont étroitement liés. Il écrit en effet :

[...] Leibniz a compris la nécessité de réformer la mécanique cartésienne et de substituer au principe de conservation de la quantité de mouvement (mesurée par le produit de facteurs $m v$ ) un nouveau principe de conservation, qui s'identifiera à la force vive (mesurée par le produit de facteurs $\left.m v^{2}\right)^{9}$

Ensuite, il affirme :

Selon Leibniz, il est nécessaire de postuler des entités théoriques dont le concept représente des propriétés essentielles des corps par delà la grandeur extensive et l'impénétrabilité. Ces entités théoriques doivent pouvoir rendre compte des propriétés phénoménales, plus ou moins géométriquement exprimables. À ces entités théoriques, raison suffisante de type ontologique et causal, s'articulent des « lois métaphysiques » qu'il convient d'ajouter aux « lois de l'étendue ». De la combinaison des deux types de lois naissent des a règles systématiques du mouvement. "Ces lois métaphysiques se ramènent aux principes de continuité, de l'égalité de l'action et de la réaction, de l'équivalence entre cause pleine et effet entier, de la conservation de l'efficience de la force dans le tempis sans ampliation ni destruction. [...] En postulant la force vive intrinsèque par delà les caractéristiques extensives des réalités corporelles, il s'agit de se donner la raison d'appliquer aux phénomènes physiques des vérités d'un autre ordre que les seuls axiomes logiques et géométriques, lesquels sont relatifs « au grand et au petit, au tout et à la partie, à la figure et au situs ». Ces vérités ou principes concernent le rapport de causalité, le rapport d'équivalence de l'action et de la passion, et ils servent à garantir rationnellement l'ordre des choses ${ }^{10}$.

En effet, pour pouvoir apprécier toute la portée du nouveau point de vue développé par Leibniz, il est capital de comprendre ce qui en fait la différence par rapport à la conception cartésienne. Le point de départ de la critique de Leibniz à la physique de Descartes est la notion de «quantilé de mouvement ", qu'il rejette. C'est une critique qui revient dans plusieurs écrits de

9. F. Duchesneau, op. cit., p. 100.

10. Ibid., p. 234-35 
Leibniz. Par exemple, dans Essay de dynamique sur les lois du mouvement, qui fait partie du corpus de textes formant la Dynamique, Leibniz écrit tout au début :

L'opinion que la même Quantité de Mouvement se conserve et demeure dans le concours des corps, a régné longtemps, et passait pour un Axiome incontestable chez les Philosophes modernes. On entend par la Quantité de Mouvement le produit de la Masse par la vitesse, de sorte que la masse du corps étant comme 2 et la vitesse comme 3, la quantité de mouvement du corps serait comme 6 . Ainsi s'il y avait deux corps concourants, multipliant la masse de chacun par sa vitesse et prenant la somme des produits, on prétendait que cette somme devait être la même avant et après le concours. [...] Pour revenir donc à ce que je dis de la conservation de la Force absolue, il faut savoir que l'origine de l'erreur sur la Quantité de Mouvement vient de ce qu'on l'a pris pour la Force. On était porté, je crois, naturellement à croire que la même Quantité de la Force totale demeure avant ou après le choc des corps, et j'ai trouvé cela très véritable. Or la Quantité de mouvement et la Force étant prises pour une inême chose, on a conclu que la quantité de mouvement se conservait. Ce qui a contribué le plus à confondre la Force avec la Quantité de Mouvement, est l' abus de la doctrine Statique, que deux corps sont en équilibre, lorsqu'en vertu de leur situation leurs vitesses sont réciproques à leurs masses ou poids, ou quand ils ont la même quantité de mouvement $^{11}$.

Mais, à vrai dire, cette réciprocité ou égalité de la quantité de mouvement n'a lieu que dans le cas de la « force morte » ou, comme Leibniz le dit, « du mouvement infiniment petit " qu'il appelle également "sollicitation " ${ }^{12}$, mais un tel mouvement n'est pas encore actif. C'est qu'il faut distinguer entre force morte et force vive: la première n'est sujette qu'à des sollicitations (conatus) ; la seconde est susceptible d'impétuosité (impetus). Dès qu'on a

11. Cf. G.W. Leibniz mathematische Schriften, ( ${ }^{\mathrm{re}}$ éd., 1860), édités par C. I. Gerhardt, vol. VI : Dynamica, Georg Olms Verlag, Hildesheim/New York, 1971 (p. 215-218 sq.). Voir aussi, « Brevis demonstratio erroris memorahilis Cartesii et aliorum circa legem naturalem, secundum quam volunt a deo eandem semper quantitatem motus conservari, qua et in re mechanica abutuntur» (1686), in : Gottfried Wilhelm Leibniz philosophische Schriften, vol. IV : Schriften zur Logik und zur philosophischen Grundlegung von Mathematik und Naturwissenschaft, hrsg. von C.J. Gerhardt (1875-1890), nouv. éd., par H. Herring, Darmstadt, Wissenschaftliche Buchgesellschaft, 1992, p. 219 ; et « Remarques sur la partie générale des principes de Descartes » (1692), in : G.W. Leibniz. Opuscules philosophiques choisis, traduits du latin par P. Schrecker, Vrin, Paris, 1978, p. 33.

12. « J'appelle sollicitations, écrit Leibniz, les efforts infiniment petits ou conatus, par lesquels le mobile est sollicité ou invité, pour ainsi dire, au mouvement, comme est par exemple l'action de la pesanteur, ou de la tendance centrifuge, dont il en faut une infinité pour composer un mouvement ordinaire. », in : Dynamica, op. cit., p. 234. 
conçu la force vive ou l'impétuosité, « les hauteurs auxquelles ces corps pourraient arriver, ne sont point proportionnelles aux vitesses, mais comme les carrés des vitesses. Et c'est pour cela qu'en cas de force vive les forces ne sont point comme les quantités de mouvement ou comme les produits des masses par les vitesses. "Quoique l' impetus corresponde à première vue à une simple sommation de conatus, en réalité il introduit une quantité intensive (l'intensio), c'est-à-dire une nouvelle dimension dans le passage de la puissance à l'effet du mouvement, qui s'exprime par le facteur $v$ de la vitesse. En effet, tandis que la quantité de mouvement ou force morte s'épuise dans l'instant même du mouvement actuel, lorsque celui-ci arrive à terme en atteignant, par exemple, un état de repos ou d'équilibre (ce qui arrive dans le cas du mouvement inertiel), la force vive ou l'action motrice, au lieu de s'évanouir, se transforme ou se reproduit en fonction de l'espace, suivant les vitesses au carré et par rapport au temps. Il faut donc déjà distinguer entre le simple facteur constant $m v$, et le facteur intensif $v$, ce qui d'ailleurs permet de se représenter une variation du produit nivv suivant l'accélération $\gamma$. Cela fait dire à Leibniz qu'il a

[...] dévoilé les sources de la science dynamique relative à la nature de la puissance et de l'action, source que l'on n'avait pas assez explorées jusqu'alors [...] par l'établissement du principe très simple et très général de l'égalité entre cause et effet, d'où nous déduirons certes toutes les merveilles de la nature par un traitement particulier ${ }^{13}$.

Dans les mouvements envisagés par la mécanique cartésienne et dans le cas plus général du mouvement rectiligne uniforme, l'accélération est nulle, la vitesse constante et l'impulsion ou élan peut être également mesuré par rapport à l'espace ou par rapport au temps : $\gamma=0, v=$ const., $x=v t=$ const. $\times t$. Mais il ne s'agit que d'un cas particulier. Le conatus ne saurait alors y avoir un sens bien déterminé. Pour faire ressortir le passage au cadre de la dynamique leibnizienne, considérons le cas plus général de la chute des corps. Ici, l'accélération est constante, les espaces ne sont plus proportionnels aux vitesses, mais à leurs carrés :

$$
\begin{gathered}
x=\int_{t_{o}}^{t} \frac{d x}{d t} d t=\int_{t_{o}}^{t} \frac{\gamma t^{2}}{2} \\
v^{2}=\gamma^{2} t^{2}=\gamma\left(\gamma^{2}\right)=2 \gamma x \\
v^{2}=2 \gamma x ; \quad \mathrm{v}_{1}^{2}=2 \gamma x_{1} \\
\frac{v^{2}}{v_{1}^{2}}=\frac{2 \gamma x}{2 \gamma x_{1}}=\frac{x}{x_{1}}
\end{gathered}
$$

13. In : « Dynamica de potentia », Leibniz mathematische Schriften, op. cit., p. 464. 
Dans le cas du mouvement uniforme, tout se passe comme si la somme des impulsions pouvait être exprimée indifféremment par $\Sigma_{m v}$ ou par $\Sigma_{m x}$. Les deux formules sont en fait équivalentes dans ce cas. Dans le cas du mouvement uniformément accéléré, la mesure de la force par l'espace conduit non pas à la formule de la quantité de mouvement, mais à celle de la force vive :

$$
m x=\int_{t_{o}}^{t} \frac{d x}{d t} d t=m v^{2} \quad \text { (puisque } v^{2} \text { est comme } x \text { ). }
$$

Il convient donc de réexaminer les notions de conatus et d'impetus à la lumière de cette dernière équation. Le conatus est la différentielle de vitesse : $\gamma d t$, où $\gamma$ peut à sont tour être fonction du temps. On peut écrire l'accélération comme dérivée seconde de l'espace par rapport au temps. Ainsi la formule du conatus est la suivante :

$$
\gamma d t=\frac{d^{2} x}{d x^{2}} d t
$$

La distinction faite par Leibniz entre quantité de mouvement (force morte) et action motrice (force vive) et l'autre qui en découle entre conatus et impetus, de même que la mise en évidence d'une vis viva à l'intérieur même de l'impetus, constitue l'intuition fondamentale de sa Dynamique. À propos d'une telle distinction, Duchesneau écrit :

Du point de vue physique, cela [c'est-à-dire la loi leibnizienne de la force vive] peut se représenter comme suit : l'effet intégral de la puissance se construit par la diffusion de l'impetus; la vitesse crée alors un doublement de la proportion d'effet dynamique ; et ce paramètre se conjugue à celui de la masse et de la vitesse intensive. [...] Dans le concours des corps, au moment de l'impact, la diffusion se résorbe, et tout se ramène ì une compensation des effets dynamiques suivant les éléments de masse et les conatus élémentaires s'y rapportant. Mais la force vive peut se réengendrer compte tenu des conditions d'élasticité qui prévalent dans la structure des corps en présence. C'est pourquoi, mis à part le strict équilibre des impetus, dans tous les autres cas, la force vive se manifeste de nouveau après le choc par des effets dépassiant le stade de mouvements embryonnés et contraints de rester tels. Le repos n'est dans le cas d'équilibre qu'une apparence recouvrant une somme indéfinie de mouvements virtuels, ce que Leibniz montre excellemment par sa théorie de l'impetus. ${ }^{14}$

Plus tard, ce sera la différentielle qui, en tant que nouvel objet mathématique introduit par Leibniz lui-même dans un autre contexte, prendra complètement en charge la signification à la fois physique et mathéinatique du concept de force motrice ou d'accélération. En fait, il semble impossible de comprendre pleinement la véritable signification de la conception de Leibniz sans voir le lien étroit et en quelque sorte nécessaire entre la loi de la force

14. F. Duchesneau, op. cit., p. 202-203. 
vive et la différentielle et, plus généralement, le principe de continuité. La différentielle sert à rendre compte de l'écart qualitatif irréductible qui existe entre le mouvement conçu comme sommation de l'ensemble des sollicitations, du mouvement capable de recevoir de nouvelles intensités et par là des gradations autres provenant de la force vive s'étant engendrée en fonction de l'espace et en raison double du temps. La loi selon laquelle nous additionnons les mouvements infiniment petits pour obtenir l'effet final n'est donc pas du même ordre que la loi différentielle ou intégrale par laquelle on reconsidère les différences intervenues dans les vitesses de même que les effets globaux. Loin d'avoir une fonction purement symbolique ou de simple représentation mathématique, la différentielle sert au besoin fondamental d'exprimer l'émergence d'une nouvelle force, à savoir la vis viva ${ }^{15}$. Cela reflète d'ailleurs

15. À ce sujet, cf. l'intéressante analyse de J. Vuillemin dans Physique et métaphysique kantiennes (1955), Paris, P.U.F., 1987, en particulier p. 216-231. L'auteur y souligne que l'erreur de Descartes dans la manière de considérer le concept de vitesse aurait consisté essentiellement à traiter des quantités vectorielles comme des quantités scalaires, en contredisant ainsi la vraie nature du mouvement et en rendant inintelligible la notion même de conservation. Huyghens et Leibniz ont vu cette erreur, et ils ont compris que le principe cartésien de la conservation du mouvement n'est vrai que pour des cas tout à fait particuliers; il n'a de valeur générale que si on tient compte des signes des vitesses, c'est-à-dire si on se fait du théorème de la quantité de mouvement une conception vectorielle et non plus scalaire. La critique de Huyghens et Leibniz « a pour conséquence d'interdire qu'on conçoive la quantité de mouvement [...] comme un absolu. Si cette quantité est vectorielle, s'il faut tenir compte du signe des vitesses pour que la formule soit générale, le mouvement n'étant alors, selon la conception cartésienne qu'un changement de position, c'est-à-dire quelque chose de relatif, il faut donner un sens relatif à la formule de conservation et non un sens absolu. C'est bien ainsi que Leibniz interprète Descartes, car si le principe essentiel qu'il introduit est celui de la conservation des forces vives, il ne laisse pas de lui ajouter trois autres principes de conservation... » Leibniz appelait la conservation de la force absolue ou action motrice le produit de la quantité de mouvement par l'espace parcouru ou de la force vive par le temps, puisque : $m v s=m v . v t=m v^{2} t$, c'est-à-dire : $a v v+b y y$ $=a x x+b z z$, qui est l'équation donnée par Leibniz. "Cette équation, remarque Leibniz, a ceci d'excellent que toutes les variations de signes qui ne peuvent venir que de la diverse direction des vitesses $y, x, z$, cessent parce que toutes les lettres qui expriment ces vitesses montent au carré. [...] Et c'est aussi pour cela que cette équation donne quelque chose d'ahsolu, d'indépendant des vitesses respectives ou des progrès d'un certain côté. Il ne s'agit ici que d'estimer les masses et les vitesses, sans se mettre en peine de quel côté vont ces vitesses. " (cité de Essay de Dynamique sur les loix du mouvenent..., op. cit., p. 227-228.) Vuillemin donne de ce passage de Leibniz un commentaire particulièrement intéressant : "La recherche de l'absolu, de ce qui se conserve immuable dans le mouvement, ne peut être menée à bien que si, dans le mouvement lui-même, qui est par essence relatif, on découvre quelque chose qui ne soit pas affecté par cette relativité. Descartes commet l'erreur de chercher cet absolu dans une quantité dont la signification est déterminée par le sens relatif des vitesses : on voit pourquoi sa tentative aboutit nécessairement à une contradiction. La signification vectorielle de la quantité de mouvement suffit à montrer que tout espoir d'identifier cette quantité avec une substance 
une loi profonde de la nature : les corps soumis à un mouvement en plus de dépenser de la force ou de l'action, peuvent également en créer, ou bien encore transformer un certain type d'action en un autre type d'action. Reprenons les considérations intéressantes développées par Gilles Châtelet à ce sujet :

C'est ainsi que la masse (simple facteur d'inertie chez Descartes) devient élastique et permet désormais l'acquisition progressive de force vive, ne réduisant pas l'information de la matière au gain brutal d'impulsion par chocs. Le concept de force vive est explicitement reconnu par Leibniz comme situé à la charnière de l'articulation du physique et du mathématique. Cette force vive n'est pas " géométrique ". Elle ne surgit pas de la Géométrie par des déductions purement mathématiques. Elle est purement physico-mathématique au sens où la connexion causale qui lie les différents instants (et appréciée par l'élément de force vive) est à identifier avec la synthèse effectuée par la Différentielle entre l'espace attaché à l'instant t et l'instant $t+D t$. C'est bien une extension radicale du projet galiléen [et cartésien] : la Forre vive importe le monde des Formes limites de la Géométrie pure, par dessus les figures et les identités, pour rencontrer les connexions causales du monde des corps. Chez Leibniz, l'émergence d'un nouveau type d'être mathématique, la Différentielle, est tout à fait contemporaine de l'idée d'élément de force vive qui permet de saisir le conatus (l'accélération) avant toute actualisation par impétuosité. L'intégrale des éléments de force vive permet de lier le passé du corps tout en enveloppant l'avenir ${ }^{\text {lt }}$.

Par ailleurs, la loi de continuité est aussi un concept-clé de la science et de la métaphysique leibniziennes, et ce, au moins au double titre de principe heuristique et de principe de justification de la cohérence de la théorie, notamment de sa Dynamique. Par principe de justification, il ne faut pas simplement entendre le fait qu'il permet de s'assurer du bien-fondé de la théorie physique par une analyse a posteriori de la compatibilité formelle des résultats de cette demière relativement à certains axiomes fondamentaux posés au préalable. Certes, aucune théorie physique ne peut prétendre violer le principe de continuité, car il est un principe de raison en quelque sorte nécessaire comme il y en a peu d'autres. Mais en même temps, il est un principe métaphysique dans la mesure où il exprime un niveau sous-jacent à l'explication des phénomènes physiques en tant que tels. Autrement dit, tout en étant un principe de nature mathématique, il est aussi d'une certaine manière ontologiquement déterminé. En tout cas, il n'y a pas de doute qu'il fait partie

métaphysique était vain par principe. On comprend aussi pourquoi la recherche de la substance, de l'absolu qui se conserve, ne peut aboutir pour Leibniz, que si l'on dépasse l'existence relative des mathématiques et que si l'on découvre une grandeur permanente scalaire et par conséquent indépendante des définitions relatives propres aux déplacements géométriques » (ibid., p. 225).

16. G. Châtelet, Les enjeux du mobilé. Mathématiqué, physique, philosophie, Seuil, Paris, 1993, p. 50-51 sq 
constitutive de la physique et de la conception que Leibniz a cherché à en développer dans la Dynamique. D'ailleurs, il tient à affirmer cela avec grande force et dans différents contextes. Voici d'abord ce qu'il écrit à ce propos en relation à l'idée de l'élasticité des corps et à leur différence qui tient de ce qu'ils sont durs ou mous, solides ou fluides :

Or cette Élasticité des corps est nécessaire à la Nature, pour obtenir de grandes et belles lois que son Auteur infiniment sage s'est proposé, parmi lesquelles ne sont pas les moindres, ces deux Lois de la Nature que j'ai fait connaître le premier, dont la première est la loi de la conservation de la force absolue ou de l'action motrice dans l'univers avec quelques autres conservations absolues nouvelles qui en dépendent et que j'expliquerais un jour, et la seconde est la loi de la continuité, en vertu de laquelle entre autres effets, tout changement doit arriver par des passages inassignables et jamais par sauts. Ce qui fait aussi que la nature ne souffre point des corps durs non-élastiques ${ }^{17}$.

De là Leibniz se sent autorisé à rejeter toutes les raisons invoquées pour donner un fondement scientifique aux phénomènes de choc car ils sont contraires à la loi de continuité. À ce propos, il s'imagine l' « expérience mentale » suivante. Il imagine un choc entre deux corps (deux globes) durs nonélastiques dont un est au repos. Deux conséquences sont alors possibles : soit qu'après le choc les deux corps sont dans un état de repos, mais dans ce cas la loi de conservation de la force vive serait violée ; soit que le choc imprime du mouvement au corps qui se trouvait au repos, mais il faudrait alors que l'autre corps (pour conserver sa force) éprouve un mouvement soudain vers l'arrière. Mais il faut exclure un tel mouvement, car il se ferait par sauts. Donc, soit que l'on suppose que "le corps choquant passe tout d'un coup de la vitesse au repos, ou bien que l'on imagine que le corps choqué passe tout d'un coup du repos à un certain degré de vitesse, dans les deux cas la loi de continuité serait violée car elle n'admet aucun changement par saut dans la nature. » Or, puisque la loi de continuité correspond mathématiquement au fait d'exprimer les lois physiques des phénomènes dans la nature au moyen du calcul différentiel (la « Differentielle » exprime précisément le fait qu'une fonction se développe par des accroissements infiniment petits), il apparaît impossible d'étudier les propriétés des phénomènes sinon conformément à la loi de continuité, qui, par conséquent, n'a pas seulement un statut mathématique, mais également physique, ou plutôt «physico-Inathématique». D'ailleurs, dans une lettre à Varignon «Sur le principe de continuité » (1702), Leibniz écrit :

[...] je me contenterai de répondre à l'article de votre Lettre, où vous me demandez des éclaircissements, sur mon Principe de Continuité. Assurément je pense que ce Principe est général, et qu'il tient hon, non seulement dans la Géométrie, mais encore dans la Physique. La Géométrie n'étant que la science des limites et

17. In : Specimen dynamicum, op. cit., p. 228-29. 
de la grandeur du Continu, il n'est point étonnant, que cette loi s'y observe partout : car d'où viendrait une subite interruption dans un sujet, qui n'en admet pas en vertu de sa nature ? Aussi savons-nous bien, que tout est parfaitement lié dans cette science, et qu'on ne saurait alléguer un seul exemple, qu'une propriété quelconque y cesse subitement, ou naisse de même, sans qu'on puisse assigner le passage intermédiaire de l'une à l'autre, les points d'inflexion et de rebroussement, qui rendent le changement explicable ; de manière qu'une Équation Algébrique, qui représente exactement un état, en représente virtuellement tous les autres, qui peuvent convenir au même sujet. L'universalité de ce Principe dans la Géométrie m'a bientôt fait connaître, qu'il ne saurait manquer d'avoir lieu aussi dans la Physique : puisque je vois, que, pour qu'il y ait de la règle et de l'ordre dans la $\mathrm{Na}$ ture, il est nécessaire, que la Physique harmonise constamment avec le Géométrique... Selon moi tout est lié dans l'Univers, en vertu de raisons de Métaphysique de manière que le présent est toujours gros de l'avenir, et qu'aucun état donné n'est explicable naturellement, qu' au moyen de celui, dont il a été précédé immédiatement. Si on le nie, le monde aura des hiatus, qui renversent le grand Principe de la Raison suffisante, et qui obligeront de recourir aux miracles, ou au pur hasard dans l'explication des Phénomènes. [...] Le Principe de Continuité est donc hors de doute chez moi, et pourrait servir à établir plusieurs vérités importantes dans la véritable Philosophie, laquelle s'élevant au-dessus des sens et de l'imagination, cherche l'origine des Phénomènes dans les Régions intellectuelles. Je me flatte d'en avoir quelques idées, mais ce siècle n'est point fait pour les recevoir ${ }^{18}$.

\section{Le rapport de Leibniz à la physique mathématique de Galilée et de Newton}

Pour Galilée et puis Newton, la physique mathématique devait se constituer hors de la Métaphysique. Il lallait pour cela libérer l'espace physique de toute propriété substantielle et en particulier penser le mouvement hors de la nature des corps. Cela supposait l'invention de l'Espace abstrait, simple aptitude à recevoir la matière et capacité d'absorber dans son homogénéité le monde des formes et celui des corps. Le projet galiléen de considérer le monde physique comme Universum mathematicum peut se comprendre comme la mise en évidence numérique de la correspondance entre la genèse des concepts géométriques et celle des actes de remplissement matériel et des connexions de causalité. Un espace physico-mathématique permet en particulier ce qu'on appelle désormais des Gedankenexperimente ${ }^{19}$, appliqués

18. In : Leibniz philosophische Schrifien, vol. IV : Schriften zur Logik und zur philosophischen Grundlegung von Mathematik und Naturwissenschaft, op cit., p. 260 $s q$.

19. Au sujet du rôle qu'auraient joué les « expériences mentales » dans la formation de la physique classique galiléenne, voir les deux interprétations divergentes 
méthodiquement par Galilée à la description mathématique des phénomènes physiques (plan incliné, mouvement d'une bille, chute des graves, etc.) et la prévision de nouvelles expériences. Par l'intermédiaire des paramétrisations et des situations idéales, ces expériences de pensée permettent de dévoiler les liaisons entre "grandeurs » mathématiques et "événements » physiques. N'est-il pas vrai qu'au fond le langage de la nature (les propriétés et les qualités des phénomenes réels) sont écrits dans une langue mathématique formée de notions abstraites, d'équations et de formules analytiques, figures et diagrammes géométriques, etc. ${ }^{x)}$ ? Comme Husserl l'a écrit :

[...] Jans la mathématisation galiléenne de la nature désormais c'est la nature même qui, sous la direction de la nouvelle mathématique, se trouve idéalisée : elle devient elle-même, pour utiliser une expression moderne, une multiplicité mathématique. [...] L'ensemble de la méthode a par avance un sens général, quand bien même ce à quoi l'on à affaire demeure toujours de l'ordre de la factualité individuelle. Par exemple, ce que l'on a d'avance en vue n'est pas la chute libre de ce corps, mais la factualité individuelle est exemplairé de la typique d'ensemble de la nature donnée à l'intuition, dans l'invariance empirique familière de laquelle il est a priori co-inclus; et cela se reporte naturellement dans l'attitude mathématico-idéalisante de Galilée. La mathématisation indirecte du monde, qui se déroule alors comme objectivation méthodique du monde de l'intuition, produit des formules numériques générales, qui, une fois trouvées, peuvent servir dans l'application à accomplir l'objectivation factuelle des cas singuliers qui doivent être subsumés sous elles. Il est manifeste que les formules expriment des liaisons causales

données par E. Mach, Die Mechanik in ihrer Entwickelung historisch-kritisch dargestellt, Leipzig, 1883, en part. les chap. 2 et 3 (éd. française, 1904) ; et par E. Cassirer, Substanzhegriff und Funktionsbegriff, Berlin, 1910 (éd. française, Les Éditions de Minuit. Paris, 1967). Ce dernier, en particulier, écrit : « Il est hors de doute pour Galilée que le principe [d'inertie], dans le sens où il le prend, ne relève pas de la considération d'une classe particulière de mouvements ayant une réalité empirique. [...] L'espace ahsolu et le temps absolu de la mécanique n'impliquent pas plus d'énigme existentielle que ce n'est le cas avec le nombre pur de l'arithmétique ou avec la ligne droite de li géométrie. Ils s'inscrivent dans le droit fil de ces concepts; et n'est-ce pas Galilée qui souligne avec la dernière vigueur que la théorie générale du mouvement représente à ses yeux une branche, non de la mathématique appliquée, mais bien de la mathématique pure ? Les concepts phoronomiques de mouvement uniforme et uniformément accéléré ne retiennent d'emblée nul trait de la constitution sensible des corps matériels; ils ne font que stipuler une certaine relation entre grandeurs spatiales et grandeurs temporelles, produites conformément à un principe génératif tout imprégné d'idéalité et mises en relation entre elles. Aussi nous suffira-t-il, pour exprimer le principe d'inertie, de nous appuyer sur un système de référence produit par la pensée et auquel nous imputerons toutes les déterminations désormais requises $\gg$ (p. 206 et 213).

20. Cf. G. Galilée, Il Saggiatore (1623), et Discorsi e dimostrazioni matematiche intorno a due nuove scienze (Leyde, 1638), in : Opere di Galileo Galilei, Édition Nationale, tome VI et VIII, Florence, 1890-1909. 
générales, des « lois de la nature ", des lois de dépendance réelle, sous la forme de la dépendance « fonctionnelle » des nombres ${ }^{21}$.

La mécanique mathématisée de Galilée se fonde sur deux concepts fondamentaux. D'une part, il y a l'espace-temps ${ }^{22}$, cadre naturel où ont lieu les événements du monde réel, qui est la simple combinaison de notre espace tridimensionnel et de la quantité unidimensionnelle qui est le temps. D’autre part, il existe des systèmes de coordonnées (dits inertiels) qui satisfont aux deux propriétés suivantes : i) toutes les lois de la nature, en chaque instant, sont les mêmes dans tous les systèmes inertiels de coordonnées ; ii) tous les systèmes de coordonnées qui se déplacent par rapport à un système inertiel d'un mouvement rectiligne uniforme sont aussi inertiels. L'espace-temps galiléen possède une certaine structure de groupe qui est conservée par les transformations qui le composent. Ces transformations sont brièvement :

- le mouvement uniforme avec une certaine vitesse $v$ assignée au départ,

$g_{1}(t, x)=(t, x+v t), \quad \forall t \in R, \quad x \in R^{3} ;$

- la translation de l'origine des coordonnées,

$$
g_{2}(t, x)=(t+s, x+s), \quad \forall t \in R, \quad x \in R^{3} ;
$$

- la rotation des axes de coordonnées,

$$
g_{3}(t, x)=(t, G x), \quad \forall t \in R, \quad x \in R^{3} \text {, où } G: R^{3} \rightarrow R^{3}
$$

21. In: La crise des sciences européennes et la phénoménologie transcendantale, trad. de l'all. et préface de G. Granel, Paris, Gallimard, 1976, p. 27 et 48. À ce sujet, voir I. Mittelstrab, “Galilei als Methodologe ", Berichte zur Wissenschaftsgeschichte, 18 (1995), p. 15-25. Pour une analyse intéressante de cette question, cf. P. Kerszherg, "Of Exact and Inexact Essences in Modern Physical Science », in : Phenomenology of Natural Science, L. Hardy and L. Einhree (éds.), Netherlands, Kluver Academic Publishers, 1922, p. 93-118.

22. En introduisant la loi d'inertie (qui à elle seule suffit à définir la structure affine de l'espace-temps), on obtient l'espace-temps de la dynamique classique que nous appelons aujourd'hui "l'espace-temps "spécial" non-relativiste ". Son groupe d'automorphismes, le groupe de Galilée $\mathcal{G}$ comprend le groupe élémentaire de l'espace-temps newtonien $\mathcal{F}$ qui est le produit direct du groupe des mouvements, rotations et translations de l'espace euclidien $E$ et du groupe affine de $T$, mais qui, à son tour, est inclus dans le groupe d'uutomorphismes de l'espace-temps leibnizien, appelé le groupe cynématique $\mathcal{R}$ de sorte que $\mathcal{F} \subset \mathcal{G} \subset \mathcal{R} G$ est non seulement le groupe de symétries de l'espace-temps de la dynamique classique nonrelativiste, mais encore le groupe d'invariance des lois de la mécanique qui gouverne des systèmes isolés de particules (ou des corps rigides). Pour une analyse des trois types d'espace-temps du point de vue de la physique et de la théorie modernes de l'espace-temps, $c f$. J. Ehlers, "The Nature and Structure of Spacetime ", in : The Physicist's Conception of Nature, Edited by J. Mehra, D. Reidel, Dordrecht, 1973, p. 71-91 ; et R. Penrose, The Emperor's New Mind, Oxford, Oxford University Press, 1989, p. 162-170. 
est une transformation orthogonale ${ }^{23}$.

Or tout mouvement de point matériel (inertiel) isolé et de système de points (inertiel) doit être invariant par rapport au groupe galiléen de transformations de l'espace-temps.

Avec Newton, on assiste à une radicalisation encore plus grande de ce schéma abstrait. Puisque les mathématiques et la géométrie (et les calculs qu'elles permettent de faire) sont à même de traduire au moyen de formules analytiques (quantitatives) exactes les relations entre les corps physiques ainsi que d'exprimer la quintessence des lois générales de la nature, les phénomènes (c'est-à-dire les lois du mouvement des points et systèmes matériels) doivent pouvoir se déduire par des hypothèses ad hoc. Les mathématiques ont ainsi, d'après Newton, ce pouvoir de garantir un accord complet entre les figures abstraites et les calculs analytico-algébriques et les lois des phénomènes observables.

Leur rôle doit se limiter à décrire et à prédire ces derniers, mais elles ne peuvent pas prétendre expliquer quoi que ce soit de leur nature et des principes qui Ies occasionnent. Autrement dit, les mathématiques, tout en s'appliquant aux phénomènes, gardent un rapport complet d'extériorité face aux causes proprement physiques de ces mêmes phénomènes.

\section{Le débat entre Leibniz et Newton sur l'espace et le temps absolus}

Newton et ses adeptes ont constamment affirmé (voir à ce propos la correspondance, sur laquelle nous reviendrons dans un instant, entre Leibniz et Clarke $^{24}$, un ami et porte-parole de Newton) que dans la nature il n'existe qu'un mouvement réel (ou absolu) et que la réalité de ce mouvement prouverait la réalité de l'espace absolu. Il ne serait alors question d'accorder aux corps une autre réalité que celle qui en fait de purs points géométricomatériels dans un espace abstrait et vide ; et surtout, il n'y aurait aucun sens à leur attribuer des propriétés physiques intrinsèques, des forces substantielles ou consubstantielles. En fait, tout ce qui nous est connu de la matière se réduit, d'après Newton, à un principe très simple qui est le suivant : tous les mouvements d'un système formé de $n$ points matériels (dans un système de

23. Pour un exposé complet, cf. V.I. Arnold, Méthodes mathématiques de la mécanique classique, Moscou, Éditions Mir, 1976. Pour des remarques éclairantes sur cette question, $c f$. H. Poincaré, "Le mouvement relatif et le mouvement absolu ", in : La Science et l'Hypothèse, Paris, Flammarion, 1912, p. 129-137.

24. "Recueil de lettres entre Leibniz et Clarke, 1715-1716", in : G.W. Leibniz Opera Philosophica, ergänzet und mit einem Vorwort versehen von R. Vollbrecht, Scientia Aalen, 1959, p. 746-788. À ce sujet, voir A. Koyré, Du monde clos à l'univers infini, Paris, Gallinard, 1973, en part. le chap. X. 
référence inertiel galiléen-newtonien) sont univoquement déterminés par leur position initiale, $\boldsymbol{x}\left(t_{0}\right) \in \boldsymbol{R}^{\mathrm{N}}$, et par leur vitesse initiale, $\boldsymbol{x}^{*}\left(t_{0}\right) \in \boldsymbol{R}^{\mathrm{N}}$.

La question de la nature du mouvement (absolu selon Newton, relatif pour Leibniz) et de la possibilité ou non d'admettre l'espace absolu, apparaît décisive pour comprendre les raisons du conflit apparemment inconciliable entre les conceptions de Newton et de Leibniz sur la nature de l'espace et le statut de la physique ${ }^{25}$. La polémique entre Leibniz et Newton (par l'intermédiaire de Clarke), seulement de quelques années postérieure à la conception de la Dynamique (entre 1689 et 1701), est particulièrement révélatrice; il s'agit d'un document extrêmement important pour comprendre les véritables enjeux du conflit qui sépare les deux savants. Tout semble au fond se nouer autour de la question de savoir s'il y a un sens à admettre un espace absolu et vide (de matière), indifférent aux forces, indivisible comme la matière et infini, ainsi que Newton prétendait l'affirmer ; ou bien, s'il ne faut pas reconnaître avec Leibniz que l'espace est relatif, car « il est l'ordre de ce qui coexiste ou l'ordre de tout ce qui existe en même temps ${ }^{26}$. "Dans la troisième lettre à Clarke, Leibniz précise son idée là-dessus :

Pour moi, j'ai marqué plus d'une fois que je tenais l'Espace pour quelque chose de purement relatif, comme le Temps; pour un ordre des Coexistences, comme le Temps est un ordre de Successions. Car l'espace marque en termes de possibilité, un ordre des choses qui existent en même temps, en tant qu' elles existent ensemble; sans entrer dans leur manière d'exister.

Qui plus est, puisque les corps possèdent des propriétés physiques réelles (ou des forces) en plus des simples propriétés d'étendue, et qu'ils remplissent en totalité l'espace ou quelques-unes de ses parties, il est impossible d'accorder un sens quel qu'il soit à l'idée de l'existence d'un espace vide. Leibniz affirme encore dans sa cinquieme et dernière lettre à Clarke :

Ie ne dis point que la matière et l'espace sont la même chose ; je dis seulement qu'il n'y a point d'espace où il n'y a point de matière ; et que l'espace en luimême n'est point une réalité absolue. L'espace et la matière diffèrent comme le temps et le mouvement. Cependant ces choses, quoique différentes, se trouvent inséparables.

25. Pour des considérations intéressantes sur ce point, cf. K. Meinzer, «Philosophie und Geschichte von Raum und Zeit ", in : Philosophie und Physik der Raum-Zeit, hrsg. von I. Ausdretsch und K. Mainzer, Wissenschaftsverlag, Mannheim, 1994, en part. p. 21-28; et A. Bartels, "Von Einstein zu Aristoteles. RaumzeitPhilosophie und Substanz-Metaphysik ", Philosophia Naturalis, 31, 2 (1994), p. 293-301.

26. G.W. Leibniz, Initia rerum mathematicorum metaphisica (1715), in : Leibnizens mathematische Schriften, VII, hrsg. von C. I. Gerhardt, Halle, 1863, p. 18 et sq. 
Et à un autre endroit (dans la quatrième lettre), où il est question de la conception de Newton selon laquelle le monde réel serait formé d'atomes absolument indivisibles, Leibniz écrit :

Tout de même, vouloir du vide dans la Nature (...), c'est violer le grand principe d'une raison suffisante. [...] Voici encore l'autre raisonnement pris de la nécessité d'une raison suffisante [l'autre revient à reconnaitre à Dieu le pouvoir de mettre toute perfection dans les choses]. Il n'est point possible qu'il y ait un principe de déterminer la proportion de la matière, ou du rempli au vide, ou du vide au plein. On dira peut-être que l'un doit être égal à l'autre ; mais comme la matière est plus parfaite que le vide, la raison veut qu'on observe la proportion géométrique, et qu'il y ait d'autant plus de plein qu'il mérite d'être préféré. Mais ainsi il n'y a pas de vide du tout : car la perfection de la matière est à celle du vide, comme quelque chose à rien. Il en est de même des atomes. Quelle raison peut-on assigner de bomer la Nature dans le progrès de la subdivision?

\section{La nouvelle méthodologie et la nouvelle épistémologie leibniziennes. Remarques sur quelques thèses de François Duchesneau}

Il ressort clairement de ce demier passage que le principe de raison suffisante est un principe pour ainsi dire régulateur ou a priori dont l'entrée en scène est en quelque sorte nécessaire pour conférer une intelligibilité intrinsèque à l'ordre causal des phénomènes. On peut dire la même chose de d'autres principes comme celui de continuité (tiré des mathématiques, mais dont l'application se justifie entièrement aussi en physique) et celui de conservation de la force vive, un résultat fondamental de la dynamique leibnizienne. Cependant, comme Duchesneau a tenu à le souligner, il importe de distinguer entre principes architectoniques et principes géométrico-physiques : les premiers auraient un rôle épistémologique fondamental à jouer pour la constitution théorique des structures géométriques et des hypothèses physiques de même que pour assurer un accord entre les modèles géométriques abstraits et l'ordre causal des phénomènes; les seconds opèrent au niveau de l'intelligibilité proprement physique des phénomènes et de leur constitution même. En tout cas, et c'est là une thèse centrale de toute l'argumentation développée par l'auteur de La dynamique de Leibniz, ces principes architectoniques interviennent directement et de façon essentielle, par l'intermédiaire d'entités théoriques et de modèles géométriques, dans la production théorique des phénomènes et notamment dans la constitution de la science physique des forces, à savoir la Dynamique. À ce propos, Duchesneau écrit :

La stratégie leibnizienne est d'entrée de jeu différente [de celle qui consisterait à restaurer l'architecture des substances aristotéliciennes], puisqu'elle construit beaucoup sur la représentation géométrique des phénomènes : cette représentation 
requiert une raison suffisante à la fois adéquate à l'ordre géométrique en fonction duquel les phénomènes sont représentables, et à la dérivation causale du système des phénomènes. C'est pourquoi Leibniz propose une conception théorique de la force primitive susceptible de rendre compte, par diffusion et réplication, des propriétés extensives en lesquelles les réalités phénoménales paraissent analysahles. Car la mise en forme géométrique s'impose dans l'analyse des effets : en témoigne par exemple le fait que les déplacements respectifs des corps qui se heurtent donnent lieu à des règles de conservation de la vitesse relative avant et après le choc. [...] Autrement dit, le recours aux entités théoriques doit fournir des raisons suffisantes adéquates, et donc congruentes à l'intelligibilité géométrique en laquelle se traduisent les phénomènes 27

Il y a donc un critère d'uniformité à l'intelligibilité géométrique auquel les effets du mouvement et de l'action des corps doivent satisfaire. Duchesneau continue :

Cela impose qu'au niveau des causes - celui où intervient la force comme ingrédient réel et absolu - on ait affuire à des entités théoriques dont le concept adéquat enveloppe les réquisits de l'ordre géométrique. Et ceci représente un net dépassement par rapport ì la restuuration des notions de type aristotélicien, telle celle d'entéléchie, pour représenter les entités de la théorie physique.

Mais alors, en tenant compte de ce qui vient d'être dit, n'est-il pas en un certain sens contradictoire que d'affirmer ce qui suit :

Appliquées aux phénomènes de la force, les procédures de la méthodologie leibnizienne ne valent que pour autant qu'elles servent à en inventer l'explication causale. Bien qu'elle ait nécessairement une structure hypothético-déductive, l'explication de ce type doit répondre à des critères "Logiques", qui tiennent à l'adéquation analytique des modèles "géométriquess " par rapport à l'objet ou aux objets représentés et à la convertibilité présumée des chemincments analytiques et synthétiques, "a posteriori" et "a priori ». Mais l'explication recherchée doit aussi satisfaire à des critères de type empirique et pragmatiqué : la théorie se présente comme un progranme de recherche susceptihle d'engendrer une compréhension progressivement plus intégrée et plus ample des phénomènes (nous soulignons) $)^{2 \mathrm{x}}$.

Certes, il y a des critères logiques de premier degré qu'un modèle géométrique (comme tout modèle formel) doit satisfaire. De là à affirmer que Leibniz se pose, au premier chef, un problème d'adéquation analytique des modèles géométriques, et que le type d'explication fournie par le modèle géométrique (ce à quoi, nous l'avons vu, Leibniz accorde une importance stratégique dans sa démarche épistémologique) doit satisfaire à des critères

27. F. Duchesneau, op.cit., chap. III, p. 239.

28. Ibid., « Conclusion générale », p. 346. 
empiriques et pragmatiques, il y a une grande différence. D'une manière générale (et Leibniz ne semble pas étranger à cette idée), on pourrait concevoir un modèle géométrique comme un élément indispensable à l'explication théorique d'une certaine classe de phénomènes, ce qui, en d'autres termes, permettrait d'éliminer au maximum l'arbitraire dans l'analyse de ces mêmes phénomènes et d'en caractériser par la suite les structures essentielles et communes. Une telle détermination serait fondamentalement possible grâce à certains concepts mathématiques et à une théorie analogique des équivalences qualitatives (ou à des concepts homologiques, pour utiliser une expression plus récente). De ce point de vue, il importe peu que les apparences observées des phénomènes soient en accord ou non avec le modèle géométrique. D'ailleurs, Duchesneau lui-même écrit :

Le tournant vers la dynamique s'umorce dans la Phoranomus (1689). Leibniz entreprend alors d'appliquer le principe de l'équivalence entre cause pleine et effet entier [autre principe architectonique] à l'effet formel : celui-ci sert à caractériser le mouvement comme non contraint, qui se maintient avec constance et uniformité sans production ni exhaustion de force. Pour figurer l'effet formel comme résultante de la force sous-jacente, Leihniz propose d'abord un modèle combinatoire imparfuit, qui intègre sous la mếme homologie des états substantiels et des états purement modaux. Or ce modèle est paradigmatique, car il « simule » par des définitions abstraites et une argumentation a priori la congruence présumée des causes de l'effet formel et des raisons explicatives de la force vive. L'analyse combinatoire ainsi mise en ceuvre provoque la révision et la correction des schèmes théoriques qu'elle avait initialement produits. On passe ainsi d'une science phoronomique relative à la force et à l'effet à une science dynamique relative à l'action et à la force (nous soulignons) ${ }^{29}$.

Le rapport étroit, et en quelque sorte nécessaire entre principes géométriques et entités théoriques exprimant conceptuellement un ordre causal des phénomènes, est mis en évidence par le lien qui existe entre le principe de continuité et un certain type de forces. À ce propos, Duchesneau souligne :

Sous celte exigence de raison suffisante adéquate, le recours aux forces primitives active et passive se moule suivant le principe de continuité, qui en stipule le mode d'accomplissement opératoire. [...] La connexion du principe de continuité aux notions de forces primitives constitue le fondement de la structure théorique telle que l'illustre le Specimen. Sur ce fondement on peut construire un système de conséquences constitutif de la théorie physique proprement dite ${ }^{\text {3) }}$

Pour poursuivre cette réflexion, faisons encore quelques remarques sur le principe de continuité et sur le rôle fondamental qu'il a joué dans la conception leibnizienne de la science. Ce principe exige qu'on renonce à la notion

29. Ibid, , p. 344

30. Ibid, chap. III, p. 240 . 
d'atome et donc à l'idée, défendue par le matérialisme de même que par Newton, selon laquelle les éléments ultimes de la matière sont indivisibles, une idée qui doit être vue en rapport avec la conception d'un espace amorphe et parfaitement homogène. Si l'on attribue aux corps la propriété absolue de la solidité, on ne saurait expliquer pourquoi et comment les effets physiques occasionnés par l'action réciproque des corps entre eux peuvent se transmettre continûment et progressivement d'un corps à l'autre et d'une région à l'autre de l'espace. Par conséquent, le principe de continuité doit être admis en vue de justifier la possibilité même d'une science physique des forces. D'ailleurs, cela conduit Leibniz à élargir considérablement le champ d'investigation de la physique et à passer de la considération du concept de masse à celui de force. Il peut ainsi mettre en évidence de nouvelles propriêtés physiques et d'autres types de mouvements des corps qui avaient été ignorées par une vision par trop mécanique de la nature. On ne peut en fait méconnaître que l'étude de certains phénomènes élastiques et, plus généralement, de la propriété de l'élasticité des corps ait eu un rôle important pour le programme leibnizien de constitution d'une nouvelle science des forces, d'autant plus qu'une telle propriété est tout à fait en accord avec le principe de continuité ${ }^{31}$. Ce fait a été très bien mis en relief par Duchesneau, qui écrit :

L'hypothèse générale d'élasticité dépend du principe architectonique de continuité. [...] Mais l'argument qu'il [Leihniz] présente ici est caractéristique : il généralise le concept analogique de transition graduée d'un état phénoménal à l'autre en requérant une raison suffisante qui puisse justifier quelque forme de saut que ce soit. « Mais, dans la nature [ici Duchesneau cite Leibniz], il ne peut y avoir aucun changement instantané assignable ou repérable ; et donc, d'un degré de vitesse à l'autre on ne peut passer sans degrés intemédiaires. " [...] Ce thème de l'élasticité, dont la source est au moins partiellement empirique, sert à établir le principe de la conservation relative de la vis respectiva dans le choc. Cette vis respectiva constitue la portion de potentia absoluta qui s'engendre en attribuant aux corps, dans le concours, des vitessess réciproquement proportionnelles à leurs masses $^{32}$.

L'introduction par Leibniz de l'hypothèse de l'éther (dans L'Hypothesis physica nova) doit être aussi vue dans l'optique du principe de continuité, outre le fait qu'elle sert à justifier la propagation de la lumière dans les corps qui remplissent l'espace.

31. Sur ce point précis mais d'importance, voir l'analyse pénétrante de H. Breger, «Elastizität als Strukturprinzip der Materie bei Leibniz », in : Leibniz' Dynamica, Studia Leibnitiana, Sonderheft 13, 1984, p. 112-121. Je renvoie également aux réflexions stimulantes de Gilles Deleuze dans le livre, Le Pli. Leibniz et le Baroque, Paris, Les Éditions de Minuit, 1988.

32. Op. cit. p. 205. 
Les dernières considérations montrent déjà bien, nous semble-t-il, à quel point les conceptions de Leibniz ont anticipé (faute d'un terme plus approprié) certaines idées fondamentales de la physique moderne. En effet, quoi qu'on en dise et malgré les succès expérimentaux de la science newtonienne, un certain nombre d'idées avancées par Leibniz ont été intégrées au corpus théorique de la physique et de la physique-mathématique récentes. En disant cela, on rejoint d'ailleurs ce que Duchesneau aftirme à propos de l'actualité de la pensée de Leibniz, Iorsque, par exemple, il écrit (au tout début de son livre) :

L'influence de la science leibnizienne s'est faite profondément sentir tout au long des XVIII ${ }^{\mathrm{e}}$ et $\mathrm{XIX}^{\mathrm{e}}$ siècles, alors même que régnait le paradigme newtonien. Avec le renouveau en profondeur des théories physiques depuis le début du $X X X^{\mathrm{e}}$ siècle et l'éloignement progressif du paradigme newtonien, Leihniz apparaît de plus en plus comme le promoteur d'une conception originale et à maints égards actuelle de la méthode scientifique

Cependant, notre auteur voit l'importance des découvertes leibniziennes en physique principalement dans l'intérêt de la méthodologie proposée et dans la validité de l'approche épistérnologique qui leur serait sous-jacente. Il écrit, quelques lignes plus loin :

En définitive, l'intérêt récent des scientifiques et des philosophes pour l'ceuvre leibnizienne semble surtout de nature épistémologique. Le mode de construction des théories leihniziennes retient l'attention et peut nous instruire sur des points fondamentaux de philosophie des sciences : la construction de modèles combinatoires pour l'analyse des phénomènes, les exigences de l'explication causale, la relativité des concepts de raison suffisante représentant l'ordre empirique, l'invention et la justification des théories conformément à des principes de type architectonique.

Cette thèse a élé développée davantage par Duchesneau dans son autre ouvrage récent portant sur Leibniz ${ }^{33}$. Selon l'auteur, le grand intérêt d'une étude renouvelée de ce philosophe tient d'abord à l'originalité de l'approche méthodologique de la physique qu'il développa en concomitance avec ses découvertes scientifiques majeures. L'importance de la pensée de Leibniz repose également sur le fait que son projet épistémologique peut instruire notre réflexion actuelle sur la signification des théories physiques et mathématiques récentes. À ce propos, Duchesneau semble s'écarter de l'interprétation de $M$. Gueroult ${ }^{34}$, qui, en voulant privilégier une image métaphysique de la $D y$ namique de Leibniz, aurait fini par négliger la portée méthodologique nouvelle de la science leibnizienne.

33. Cf. F. Duchesneau, Leibniz et la méthode de la science, op. cit.

34. Cf. M. Gueroult, Leibniz. Dynamique et métaphysique, Paris, Aubier-Montaigne, 1967. 
Quant à nous, on aurait tendance à penser que la valeur et l'importance des conceptions leibniziennes en physique (de même que ses idées mathématiques et métaphysiques) ont eu une portée bien au-delà de la signification purement épistémologique que l'on peut retenir de son œuvre, si bien que celle-ci présente un intérêt éminent. Déjà, il faut mentionner le fait que Leibniz a inspiré, bien que parfois seulement indirectement, la pratique et l'esprit scientifiques tout au long du XVIII et du XIX ${ }^{\mathfrak{e}}$ siècles. Il suffit de penser au cas de Günther Grassmann, qui s'était inspiré directement des idées de Leibniz pour développer sa théorie de l'extension (Ausdehnungslehre), une théorie mathématique générale des formes auxquelles on applique un certain type de "calcul " géométrico-algébrique fondé sur quelques concepts fondamentaux comme celui de "produit extérieur », et qui a donné lieu notamment à la construction de la théorie moderne des espaces vectoriels à $n$ dimensions ${ }^{35}$. Pensons également à Bernhard Riemann, lequel avait compris l'urgence d'un dépassement de la conception newtonienne de la science de manière à permettre un élargissement des concepts et des méthodes de la géométrie et de la physique-mathérnatique, et un approfondissement des connaissances de l' univers et surtout du monde de l'infiniment petit. Dans l'esprit de Riemann, la physique mathématique ne pouvait se construire qu'au point de connexion de la structure géométrique infinitésimale de notre univers et de l'ordre causal régissant le comportement des forces physiques fondamentales de la nature ${ }^{36}$. Songeons, enfin, au statut du concept de potentiel dans la dynamique hamiltonnienne, ainsi que dans certaines théories de la physique des particules élémentaires et dans la théorie des champs. Dire que ce concept représente l'accomplissement d'une idée leibnizienne paraîtra sans doute exagéré, bien que cela contienne une part de vérité. Un potentiel possède une signification géométrique précise, mais il exprime également un contenu physique fondamental; le fait est que ces deux aspects coexistent et ils sont absolument indissociables. Puisqu'il n'est pas question ici d'approfondir un tel sujet, bomons-nous à mettre en évidence ces points qui montrent, semble-til, une influence et une convergence significative des conceptions de Leibniz et d'un certain nombre d'acquis de la science moderne :

1) La cosmologie n'admet ni espace ni temps absolus : selon une des théories les plus accréditées (la théorie du Big Bang), l'univers serait né d'une

35. Sur l'(exuvre du mathématicien, voir D. Flament, « La lineale Ausdehnungslehre (1844) de Hermann Günther Grassmann », in : 1830-1930: A Century of Geometry. History, Epistemology and Mathematics, L. Boi, D. Flament, J.-M. Salanskis (éds.), Berlin/Heidelberg, Springer-Verlag, 1992, p. 205-221.

36. Pour une analyse approfondie de ce sujet, $c f$. L. Boi, Le problème mathématique de l'espace. Une quête de l'intelligible. Heidelberg/Berlin/New York, Springer, 1995, en part. les chap. III et IV. 
explosion originelle qui aurait eu lieu il y a plusieurs milliards d'années (c'està-dire d'une singularité initiale dans l'espace et le temps) ${ }^{\mathfrak{H}}$.

2) La théorie de la relativité restreinte a conduit également à rejeter toute possibilité de concevoir en termes physiques un espace et un temps absolus. On ne peut plus affirmer qu'il existe un système de repère inertiel absolu auquel on peut rapporter tous les mouvements. Le temps mesuré par une horloge dépend désormais de la vitesse de déplacement de celle-ci et on ne peut plus déterminer si des événements se produisent à différents points de l'espace : ceci à nouveau dépend du système de repère choisi. Autrement dit, il est impossible de reconnaître un sens à la notion d' " état de repos dans l'espace absolu ». Il n'y a aucun moyen, par exemple, de mesurer le mouvement de la terre à travers l'espace absolu. On ne peut mesurer la vitesse du mouvement de la Terre que relativement à un autre corps physique comme le Soleil ou la Lune. Dans toute sa généralité, le principe de relativité d'Einstein (le célèbre principe d'équivalence qu'il a énoncé en 1905) nous dit que les lois de la physique ne peuvent foumir aucun moyen de distinguer un système de référence inertiel (que l'on suppose en mouvement uniforme) de quelque autre système de référence inertiel. Toute loi physique doit ainsi avoir la même forme logique et mathématique indépendamment du sysième de référence inertiel par rapport auquel elle se trouve appréciée ${ }^{38}$.

3) La théorie de la relativité générale est fondée sur l'idée fondamentale qu'il est impossible de concevoir l'espace indépendamment de la matière qu'il contient. Plus précisément, le type de géométrie de notre espace est en quelque sorte déterminé par la nature des forces et des phénomènes physiques se déroulant en lui, et, réciproquement, la façon dont ces forces se produisent et agissent dans l'espace dépend de la structure géométrique qui le régit.

4) La théorie physique des particules élémentaires a montré que la matière n'est pas indivisible et que, par conséquent, la conception selon laquelle la matière serait formée d'éléments ultimes est dépourvue de tout fondement.

5) La théorie de jauge a mis en évidence qu'on ne peut pas penser les mathématiques comme extérieures à la physique ; elles peuvent éventuellement s'y appliquer (a priori ou a posteriori) par la suite. En un certain sens, cette théorie suggère de considérer les entités physiques comme des "objets " géométriques en acte : "Cette rencontre entre réalité physique et conceptualité mathématique est comprise comme l'acte de traduction qui identifie un rassemblement de fibrés par transport parallèle et un potentiel vecteur créateur de particules virtuelles, véhicules de l'interaction. En fait, ce qui fonde un

37. Pour un exposé complet du sujet, cf. M. Zeilik, Astronomy: The Evolving Universe, New York, Harper \& Row, Publishers, 1979, en particulier chap. 21.

38. Pour un exposé complet, cf. K.S. Thorne, Black Holes and Time Warps, W.W. Norton \& Company, New York/London, 1994, p. 72-86. Pour une tentative de comparaison entre le principe de la relativité et la dynamique leibnizienne, voir $\mathrm{C}$. Comte, « Leibniz aurait-il pu découvrir la relativité ? », European Joumal of Physics, 7 (1986), p. 225-235. 
espace de monades mathématiciennes, c'est la connexion qui définit des transports virtuels de structures entres fibres. Et ce qui fonde un espace de monades leibniziennes, c'est le potentiel vecteur créateur de quanta virtuels d'interaction. Cette identité, obtenue par dissolution du "purement géométrique " et du "purement physique ", se réfracte ensuite sous le mode du "vraiment géométrique » (la courbure) et du « vraiment physique » (le champ électromagnétique et ses forces) ${ }^{39}$."

Les incidences épistémologiques des nouvelles conceptions mentionnées sont indéniables et leur prise en compte est d'une certaine manière fondamentale à l'approfondissement de la recherche philosophique : les mathématiques ne s'appliquent pas à la physique, elles s'y impliquent. Les concepts géométriques ne sont pas seulement des instruments théoriques servant à représenter sous forme symbolique les phénomènes réels : ils sont constitutifs des phénomènes et dans certains cas, ils sont à l'origine de leur engendrement (pensons, à ce propos, au rôle que jouent les groupes et/ou les symétries dans la plupart des théories physiques). Si on se limitait à ne voir le rôle des concepts géométriques qu'en termes d'un formalisme mathématique dont l'unique fonction serait de décrire par l'intermédiaire de modeles logiques et analytiques le réel physique et de prédire de nouvelles observations et théories, on ne saurait nullement comprendre la signification et la portée éminentes des rapports intimes, que nous évoquions plus haut ${ }^{40}$, entre réalité physique et conceptualité mathématique dans la physique théorique actuelle.

Une des thèses principales, sinon la principale, de la lecture qui nous a été proposée par François Duchesneau de l'ceuvre de Leibniz peut être présentée ainsi en quelques mots. En jetant les bases d'une nouvelle science physique, la dynamique, et en la développant ensuite dans une théorie physique des forces fondée sur le principe de conservation de la force vive, non seulement Leibniz aurait dégagé de nouveaux principes méthodologiques et un point de vue épistémologique original pour l'époque, mais encore ces mêmes principes auraient guidé ses efforts en vue d'obtenir les résultats fondamentaux de sa dynamique. Certes, la thèse est d'un grand intérêt, mais elle pourrait être développée également dans une autre direction. On peut en effet se demander s'il n'y a pas lieu d'admettre, premierement, que c'est toute la philosophie de Leibniz, et en particulier sa Monadologie ${ }^{41}$, qui est issue des

39. Cf. G. Châtelet, « Le retour de li Monade. Quelques réflexions sur le calcul différentiel et la mécanique quantique ". Fundamenta Scientiae, vol. 6, ${ }^{\circ} 4,1985$, p. 327-345.

40. Pour être à même d'aborder épistémologiquement les nouvelles conceptions rappelées, il faut remettre en discussion des points de vue applicationnel et quantitatif dans la science, en développant de nouvelles perspectives théoriques dans la recherche fondamentale et dans la réflexion philosophique.

41. Cf. «Les principes de la philosophie ou la Monadologie », in : Letzte Schriften, G.W. Leibniz philosophische Schriften (1875-1890), vol. I : Kleine Schriften zur 
principes de sa dynamique, et que c'est en elle qu'elle a puisé notamment les concepts de substance et de "monade " ${ }^{42}$, et deuxièmement, que c'est toute une conception métaphysique plus générale de la nature qui est à l'cuuvre dans la constitution de la dynamique. Après tout, Leibniz commence l'étude de ce sujet philosophique par une spéculation sur la nature des corps. Il faut ajouter que dans la Monadologie Leibniz reconnaît toutes les limites d'un discours purement mathématique, ou purement physique. D'ailleurs, le concept de force, introduit d'abord par Leibniz dans ses écrits de dynamique (dans $D y$ namica de potentia ... de 1689), se transformera plus tard en celui de monade (substance ou entelechia) dans le cadre plus général d'une recherche sur les principes métaphysiques qui permettraient d'expliquer la nature essentielle de la totalité des phénomènes naturels (aussi bien du monde animé qu'inanimé). Le Système nouveau de la nature et de la communication des substances (de 1695) donne déjà des indications sur un tel passage et sur la façon dont l'auteur conçoit le rapport entre la force comme entité physique et la force comme substance de nature métaphysique. Après avoir rappelé au début que ses « essais de Dynamique ont un lien avec celui-ci [Le Système ...] », Leibniz écrit :

Mais depuis, ayant tâché d'approfondir les principes mêmes de la Mécanique, pour rendre raison des lois de la nature que l'expérience faisait connaître, je m' aperçus que la seule considération d'une masse étendue ne suffisait pas, et qu'il fallait employer encore la notion de la force, qui est très intelligible, quoiqu'elle soit du ressort de la Métaphysique. [...] je m'aperçus qu'il est impossible de trouver les principess d'uné véritable Unité dans la matière seule ou dans ce qui n'est que passif, puisque tout n'y est que collection ou amas de parties jusqu'ì l'infini. [Or la multitude ne pouvant avoir sa réalité que des unités véritables qui viennent d'ailleurs et sont tout autre chose que les points mathématiques qui ne sont que des extrémités de l'étendue et des modifications dont il est constant, que le continuum ne saurait être composé. Donc pour trouver ces unités réelles, je fus contraint de recourir à un point réel et animé pour ainsi dire, ou à un Atome de substance qui doit envelopper quelque chose de formel ou d'actif, pour faire un être complet]. Il fallut donc rappeler et comme réhahiliter les formes substantielles, si décriées aujourd'hui, mais d'une manière qui les rendît intelligibles [...] Je trouvais donc que leur nature consiste dans la force et que de cela s'ensuit quelque

Metaphysik, op cit., p. 438-483. Sur cette question, cf. M. Gueroult, Dynamique et Métaphysique leibnizienne's, Paris, Les Belles Lettres, 1934

42. Il faut hien voir à ce propos que, si la définition cartésienne du mouvement en fait une entité imaginaire et subjective, la force vive apparaît comme ce qui se conserve dans l'univers, et à cet égard elle est comme une substance par rapport au mouvement. La recherche de la substance de l'absolu [dans le mouvement] qui se conserve, ne peut aboutir, pour Leibniz, que si l'on dépasse l'existence relative des mathématiques et que si l'on découvre une grandeur permanente scalaire et par conséquent indépendante des définitions relatives propres aux déplacements géométriques. 
chose d'analogique au sentiment et à l'appétit ; et qu'ainsi il fallait le concevoir à l'imitation de la notion que nous avons de l'âme. [...] je jugeais [...] qu'il ne fallait pas employer ces formes pour expliquer les problèmes particuliers de la nature, quoiqu'elles soient nécessaires pour établir des vrais principes généraux. Aristote les appelle entéléchies premières, je les appelle peut-être plus intelligiblement forces primitives, qui ne contiennent pas seulement l'acte ou le complément de la possibilité, mais encore une activité originale ${ }^{43}$.

En fait, on peut lire plusieurs passage de la Dynamica de potentia, du Specimen dynamicum et de l'Essay de dynamique, comme la tentative de déployer non seulement une physique mais également une métaphysique de la force. Il importe d'insister sur ce point. Ces deux aspects complémentaires de l'analyse de Leibniz se résument dans la triple distinction suivante : premièrement, entre force morte et force vive, deuxièmement, entre sollicitation (conatus) et impétuosité (impetus), enfin, entre mouvements contraints ou violents et mouvements infiniment petits, représentables mathématiquement sous forme de différentiels et conformes au principe de continuité. Pour obtenir de la force vive, il ne suffit pas de définir l'impetus comme sommation des conatus (multipliée par la masse), mais il faut en plus y ajouter une nouvelle articulation, les degrés de vitesse dont seulement la différentielle est à même de rendre compte. Ces degrés de vitesse ne s'épuisent pas en même temps que le mouvement d'un corps arrive à son terme ; autrement dit, il ne s'agit pas de le voir comme de simples quantités infiniment petites (passives), mais comme de nouvelles déterminations, des éléments de la force toujours se réactualisant en fonction de l'espace et par rapport au temps. C'est précisément sur ce point que réside l'articulation entière du raisonnement de Leibniz, et ce sur quoi repose l'intuition fondamentale de sa physique.

Dans la nouvelle physique, "le conatus, devenu vraie différentielle, permet la jonction entre le repos qui n'est qu' un mouvement infiniment lent et le mouvement proprement dit. Puisque la sommation des conatus de même espèce suffit à foumir tous les degrés possibles de vitesse, il n'y a plus autant de conatus d'espèces différentes qu'il y a de mouvements possibles de vitesses différentes, máss il y en a autant que d'accélérations différentes. Dans le cas du mouvement uniforme, où les accélérations cessent de se sommer dans le même instant, et ne font que se répéter dans la suite des instants différents, le conatus redevient vitesse élémentaire, mais pour autant que ce mouvement uniforme existant révèle la présence dans le mobile d'une force vive, le conatus par sa quantité (l'impetus) reste toujours conçu comme somme d'accélérations élémentaires. La conception du conatus, comme terme d'une série, ou loi qui constitue la substance par rapport à l'accident constitué par la force vive instantanée, engendre une nouvelle notion du rapport de la

43. Cf. G.W. Leibniz, Systèmé nouveau de la nature et de la communication des substances (1695), par C. Frémont, Paris, GF-Flarnmarion, 1994, p. 66-67. 
substance à l'accident grâce à laquelle le phénomène physique se trouve rattaché à un fondement métaphysique substantiel de façon en quelque sorte immanente [...] Le caractere évanouissant [...] du conatus, la conception dynamique de la quantité de conatus ou impetus, qui fait de lui un moment résumant le passé et gros de l'avenir, établissent enfin une affinité entre le mouvement temporel et son élément infinitésimal, entre le fondement substantiel et la sommation des éléments et ses éléments eux-mêmes" ${ }^{44}$."

On a vu que, dans la conception cartésienne, l'impetus (l'élan) résultait de la sommation des conatus multipliée par la masse:

$$
m \int_{t_{\mathrm{o}}}^{t} \gamma d t=F t=m v .
$$

Cette formule, qui mesure la force dans l'élément de temps, parce qu'elle ne concerne que le mouvement relatif, reste abstraite du point de vue leibnizien. Elle ne permet pas de définir la réalité du mobile, puisque, du pur point de vue géométrique, on peut attribuer le mouvement tout aussi bien à tel sujet qu'à tel autre. Au contraire, la force vive nous livre quelque chose de substantiel et d'indépendant des signes des vitesses. Tel est le sens du carré de $\boldsymbol{v}$. Quant à la massee, l'expérience nous révèle son existence comme force passive... Mais celte «force » n'est capable que de résister au mouvement, elle ne peut jamais devenir cause de mouvement, c'est-à-dire force active. Si on définit, suivant Leibniz, l'impetus comme différentielle première de l'espace par rapport au temps, on voit immédiatement son rapport aux forces vives :

$$
\begin{array}{ll}
\text { (vitesse) } & v=\frac{d x}{d t} \\
\text { (impetus) } & {\left[\frac{d x}{d t} d t\right] m} \\
\text { (forces vives) } & m \int_{t_{0}}^{t} \frac{d x}{d t} \quad d t=m v^{2} .
\end{array}
$$

Une doctrine leibnizienne entièrement cohérente retiendra donc les définitions suivantes : le conatus est égal à $\frac{d^{2} x}{d t} d t$, l'impetus est donné par $m \frac{d x}{d t} d t$, et la force vive correspond à $m \int_{t_{\mathrm{o}}}^{t} \frac{d x}{d t} d t$. Le fait de concevoir une double intégrale et l'introduction de la force au lieu de la masse nous font donc passer de la formule cartésienne à la formule leibnizienne.

Département de Philosophie

Université du Québec à Montréal

Université de Montréal

44. Cf. M. Gueroult, Dynamique et métaphysique leibniziennes, op cit., p. 160-161. 